Annuaire suisse de politique de développement

21 | 2002

Agriculture suisse et mondialisation

\title{
La Suisse dans les nouvelles négociations agricoles de l'OMC
}

Christian Häberli

\section{OpenEdition}

1 Journals

Édition électronique

URL : http://journals.openedition.org/aspd/919

DOI : 10.4000/aspd.919

ISSN : 1663-9669

Éditeur

Institut de hautes études internationales et du développement

Édition imprimée

Date de publication : 1 avril 2002

Pagination : 5-25

ISSN : 1660-5934

\section{Référence électronique}

Christian Häberli, «La Suisse dans les nouvelles négociations agricoles de l'OMC », Annuaire suisse de politique de développement [En ligne], 21 | 2002, mis en ligne le 06 septembre 2012, consulté le 08

septembre 2020. URL : http://journals.openedition.org/aspd/919; DOI : https://doi.org/10.4000/aspd. 919

(C) The Graduate Institute | Geneva 


\section{LA SUISSE DANS LES NOUVELLES NÉGOCIATIONS AGRICOLES DE L'OMC}

\section{POINT DE DÉPART: LE DOSSIER AGRICOLE À SEATTLE}

\subsection{DERRIĖRE L'ÉCRAN DE FUMÉE: QUE S'EST-IL VRAIMENT PASSÉ À SEATTLE?}

Seattle, $3^{\mathrm{e}}$ Conférence ministérielle de l'OMC, du 30 novembre au 4 décembre 1999: des images de manifestants déchaînés et roués de coups, l'entrée en scène du mouvement international antimondialisation. On se souviendra aussi de l'absence de déclaration officielle - phénomène sans précédent dans la diplomatie du commerce international. Et l'on n'a même pas été capable, sur le moment, de lancer un nouveau cycle de négociations. Mais que s'est-il vraiment passé derrière le rideau de gaz lacrymogènes?

Il est vrai que ces manifestations avaient de quoi impressionner. Une vaste coalition antimondialisation regroupant écologistes, syndicalistes, protecteurs des animaux de tous les continents - certains pacifiques et pleins d'humour, d'autres résolus à en découdre - s'y était préparée durant des mois à l'aide d'Internet et du téléphone mobile. Il n'empêche que ce ne sont pas les manifestants qui ont fait échouer cette conférence, mais les délégations gouvernementales ellesmêmes. Elle avait déjà été mal préparée: les négociateurs s'étaient obstinés pendant des mois à Genève sur le dossier agricole, réputé le plus difficile, en négligeant de façon impardonnable leur promesse faite à Marrakech ${ }^{1}$ d'examiner la question fondamentale de l'attitude à adopter face aux disparités croissantes dans le développement des membres de l'OMC, dont le nombre dépasse actuellement 140. A Seattle même, la direction de la conférence, présidée par la déléguée américaine Helen Barshevsky, est restée concentrée essentiellement sur le dossier agricole. Et lorsqu'un compromis s'est enfin dessiné - cinq heures avant la fin de la conférence - il était trop tard pour aborder également les autres problèmes. Une centaine de pays en développement ont alors protesté vigoureusement contre cet escamotage de leurs préoccupations. Les ébauches de toute une série d'autres négociations sont également restées en plan; on n'a même pas réussi à dresser une liste commune des sujets à traiter!

\subsection{QUE VEULENT LES ACTEURS PRINCIPAUX DU DOSSIER AGRICOLE?}

Depuis le dernier ( $8^{\mathrm{e}}$ ) cycle de négociations du $\mathrm{GATT}^{2}$, les pays concernés sont pour la plupart restés sur leurs positions ${ }^{3}$ :

* Chef des affaires internationales à l'Office fédéral de l'agriculture, Berne.

Le point de vue présenté dans cet article n'engage que son auteur.

1. Conférence ministérielle clôturant le $8^{\mathrm{e}}$ (et dernier) cycle de négociations du GATT (Cycle d'Uruguay) et instituant l'Organisation mondiale du commerce (OMC).

2. Cycle d'Uruguay, 1986-1994. Cf. messages 1 et 2 relatifs au GATT du 19 septembre 1994 (94.079 et 94.080).

3. Cf. IUED (2001 : 170-176). 
1) Le groupe de Cairns ${ }^{4}$, ainsi nommé d'après la ville australienne où il a vu le jour, continue d'exiger la suppression totale des subventions à l'exportation et de toutes les subventions intérieures qui ont pour effet d'orienter la production et de provoquer des distorsions commerciales.

2) Les Etats-Unis partagent largement ce point de vue, dans la mesure toutefois où cela ne touche pas leurs propres instruments de subvention - notamment leurs mécanismes de compensation intérieure (lorsqu'il y a chute des prix du marché mondial) et leurs aides à l'exportation. La nouvelle loi sur l'agriculture des Etats-Unis prévoit en particulier des dépenses additionnelles considérables dans ce domaine.

3) Les pays pratiquant les plus forts soutiens agricoles (UE, Japon, Corée du Sud, Norvège et Suisse, puis, après Seattle, l'île Maurice) ont formé le groupe des multifonctionnalistes, soutenus en particulier par les pays en transition d'Europe centrale et orientale. Ils ne s'estiment pas en mesure de supporter un libre-échange agricole à l'échelon planétaire et demandent à l'agriculture de fournir volontairement des prestations «non agricoles», c'est-à-dire des biens demandés par la société sans être rémunérés par le marché. Selon les circonstances et les choix de société, ces prestations peuvent être la conservation de l'environnement et des paysages, une contribution à la sécurité de l'approvisionnement ou une occupation décentralisée du territoire national.

4) Les autres pays qui n'appartiennent pas au groupe de Cairns forment un groupe hétérogène avec différents sous-groupes qui se chevauchent en partie, par exemple les importateurs nets de denrées alimentaires ${ }^{5}$, les pays de monoculture $^{6}$ tributaires d'un accès privilégié à leurs anciennes puissances coloniales, et, d'une manière générale, les économies «faibles» des pays les moins avancés (PMA) ${ }^{7}$ que toute perturbation extérieure touche de plein fouet. Le dénominateur commun des pays en développement est la revendication d'un meilleur accès au marché des pays industrialisés ainsi qu'une libération de toute obligation ou restriction touchant à leur propre politique agricole ${ }^{8}$.

\subsection{QUE VEUT LA SUISSE?}

La délégation de négociation suisse - dirigée par le conseiller fédéral Pascal Couchepin, assistée pour le dossier agricole par le Secrétariat d'Etat à l'économie (Seco) et par l'Office fédéral de l'agriculture (OFAG) - a reçu un mandat relativement précis du Conseil fédéral. Précis dans le sens d'une idée claire sur ce qui est négociable en matière agricole et sur ce qui ne l'est pas.

4. Membres: Afrique du Sud, Argentine, Australie, Bolivie, Brésil, Canada, Chili, Colombie, Costa Rica, Guatemala, Indonésie, Malaysia, Nouvelle-Zélande, Paraguay, Philippines, Thaïlande et Uruguay.

5. Nombreux sont les pays tributaires d'une aide alimentaire, non seulement dans le groupe des plus pauvres, mais aussi dans d'autres régions comme l'Afrique du Nord ou l'Asie. Par ailleurs, cette aide peut constituer pour les pays fournisseurs un moyen d'écouler leurs excédents, donc une forme possible de subvention à l'exportation qu'il convient de traiter dans le cadre des négociations agricoles.

6. Il est piquant de constater que des exportateurs de sucre comme l'île Maurice, les îles Fidji ou le Malawi dépendent même des subventions à l'exportation de l'UE du fait que c'est pour eux le seul moyen d'écouler leur sucre brut sur ce marché autosuffisant!

7. Concernant le traitement spécial des PMA, cf. plus loin sous-chapitre 4.1.

8. Concernant la «boîte de développement», cf. sous-chapitre 4.2. 
La Suisse considère comme négociables tous les sujets figurant dans l'article $20^{\circ}$ de l'Accord sur l'agriculture ${ }^{10}$ de l'OMC. Cet article a déjà été adopté à Marrakech, puis approuvé par le Parlement avec l'ensemble des accords de l'OMC ${ }^{11}$. En résumé, cette "obligation de poursuivre le processus de réforme» signifie qu'à partir de 2001 il est impératif de négocier simultanément les trois grands piliers de l'accord sur l'agriculture en vigueur (accès au marché, soutien interne et concurrence à l'exportation ${ }^{12}$ ) avec l'objectif d'une libéralisation progressive du commerce agricole (à ne pas confondre avec le libre-échange!). En même temps, il faut prendre en considération les problèmes particuliers des pays en développement ainsi que les "considérations autres que d'ordre commercial» (dans l'optique suisse: les tâches multifonctionnelles de l'agriculture).

La Suisse est ainsi résolue à ne pas entrer en matière notamment sur les deux dossiers suivants: suppression totale de la protection douanière de l'agriculture et multifonctionnalité, donc sur les prestations non marchandes de l'agriculture.

La délégation suisse défend ainsi une position qui n'est pas simple, mais qui a au moins l'avantage d'être assez claire: d'une part, l'article 20 de l'Accord sur l'agriculture approuvé par le Parlement; d'autre part, les objectifs de la politique agricole suisse tels que les définit la Constitution fédérale de 1996. L'article 104 cst. prescrit à l'agriculture deux buts de valeur égale, à savoir la durabilité et l'orientation vers le marché $e^{13}$. Et notre constitution va jusqu'à spécifier les instruments à utiliser (seuls les instruments d'application importent pour l'OMC, chaque pays ayant tout loisir de fixer lui-même ses objectifs), par exemple:

- paiements directs sous réserve que la preuve soit apportée de prestations à caractère écologique;

- encouragement de formes d'exploitation en accord avec la nature, respectueuses de l'environnement et des animaux;

- prescriptions relatives à la déclaration de la provenance, de la qualité, des méthodes de production et des procédés de transformation des denrées alimentaires.

9. «Article 20: Poursuite du processus de réforme

Reconnaissant que l'objectif à long terme de réductions progressives substantielles du soutien et de la protection qui aboutiraient à une réforme fondamentale est un processus continu, les Membres conviennent que des négociations en vue de la poursuite du processus seront engagées un an avant la fin de la période de mise en œuvre, compte tenu:

a) de ce qu'aura donné jusque-là la mise en œuvre des engagements de réduction;

b) des effets des engagements de réduction sur le commerce mondial des produits agricoles;

c) des considérations autres que d'ordre commercial, du traitement spécial et différencié en faveur des pays en développement Membres et de l'objectif qui est d'établir un système de commerce des produits agricoles qui soit équitable et axé sur le marché, et des autres objectifs et préoccupations mentionnés dans le préambule du présent accord; et

d) des autres engagements qui seront nécessaires pour atteindre l'objectif à long terme susmentionné.»

10. Accord sur l'agriculture, in message 1 relatif au GATT, op. cit., p. 474.

11. Arrêté fédéral du 16 décembre 1993 (FF [Feuille fédérale] 1994, p. 540).

12. Cf. plus loin, sous-chapitre 2.3 .

13. Article 104 cst.: «La Confédération veille à ce que l'agriculture, par une production répondant à la fois aux exigences du développement durable et à celles du marché, contribue substantiellement: $a$. à la sécurité de l'approvisionnement de la population; $b$. à la conservation des ressources naturelles et à l'entretien du paysage rural; $c$. à l'occupation décentralisée du territoire.» (Etc.) 
Un tel modèle simplifie donc la représentation de la Suisse aux négociations agricoles de l'OMC: quels négociateurs peuvent se vanter d'un mandat directement issu de leur constitution nationale? Mais ce n'est qu'à «l'heure de vérité» que l'on pourra déterminer si - et dans quelles proportions - la protection douanière ou les mesures de soutien du marché pourront être diminuées ou non!

\section{DE SEATTLE À DOHA}

Comme l'article 20 susmentionné avait même fixé le début des négociations au $1^{\text {er }}$ janvier 2000, l'échec de Seattle en décembre 1999 ne pouvait plus reporter cette échéance ${ }^{14}$. Un mandat clair des ministres à Seattle aurait sans doute permis de mieux «formater» les négociations et peut-être d'en définir le calendrier. Etant donné les circonstances, il ne fallait pas songer à des pourparlers efficaces. Mais comment est-on passé du constat d'échec à la table de négociations?

\subsection{PROPOSITIONS DE LA PREMIÈRE PHASE (MARS 2000-MARS 2001)}

Une première phase de négociations courant sur une année a pu démarrer à mimars 2000 déjà ; 125 pays ont présenté 48 propositions réunies dans un tableau des points à examiner. Le contenu de ces propositions n'a rien de surprenant; il reprend les idées présentées à Seattle, quoique de façon plus détaillée. Aspect positif: pour la première fois dans l'histoire du GATT, tous les pays en développement ont alors fait des propositions. Même s'il s'agit essentiellement (comme d'ailleurs pour les propositions des pays industrialisés) de revendications et non d'offres de concessions, cela crée - avec les résultats de Doha - une base nettement plus favorable pour trouver une réponse adéquate aux besoins spécifiques du développement.

\subsection{LA PROPOSITION SUISSE}

La Suisse a également présenté une proposition détaillée pour ces négociations. Ce document a été élaboré par l'administration fédérale et, avec l'accord du conseiller fédéral compétent, soumis le 21 décembre 2001 à 1'OMC, après qu'avait été offerte aux milieux de l'agriculture, de l'industrie alimentaire, du commerce et des organisations non gouvernementales (ONG) l'occasion d'exprimer leur avis.

\section{Résumé de la proposition de la Suisse pour les négociations sur l'agriculture à l'OMC (21 décembre 2000)}

1) Conséquences des résultats du Cycle d'Uruguay: le commerce agricole mondial a augmenté depuis 1995; on peut parler d'un succès du Cycle d'Uruguay, alors même que tous les pays n'en ont pas également profité. Avec sa politique agricole 2002, la Suisse a fortement réduit son aide interne (mesures de la boîte orange) et accru simultanément les paiements au titre de la boîte verte. Les pays en développement ont même augmenté leur part des importations agricoles en Suisse dans la proportion de $20 \%$ !

14. L'engagement pris de renégocier l'Accord général sur le commerce des services (AGCS) figure à l'article XIX AGCS (RO [Recueil officiel des lois fédérales] 1995, p. 2418). 
2) Les engagements pris par la Suisse correspondent au nouvel article constitutionnel (art. 104) qui prescrit une production agricole répondant à la fois aux exigences du développement durable et à celles du marché. Tandis que la production de denrées alimentaires obéit aux lois du marché, on rémunère les prestations non marchandes (biens publics) - notamment entretien du paysage rural, occupation décentralisée du territoire ou préservation des ressources naturelles - par des paiements directs. La Suisse demande que chaque pays puisse déterminer lui-même le montant de ces paiements directs, sur la base de ses propres objectifs agricoles, environnementaux et sociétaux. Mais elle est également disposée à admettre une description précise des critères, pour éviter le reproche d'un soutien indirect à la production de denrées dont le marché peut assurer la régulation. Nos paiements directs devraient ainsi jouir d'une sécurité juridique accrue au niveau de I'OMC.

3) La Suisse exige que I'on négocie aussi dans trois domaines additionnels qui bien que traités dans d'autres accords, ont néanmoins une relation avec l'agriculture. II s'agit en premier lieu de mieux protéger les indications géographiques (appellations d'origine), non seulement pour les vins et les spiritueux mais aussi pour d'autres produits tels que les fromages. II convient ensuite de donner aux consommateurs des informations sur les produits plus complètes que jusqu'à présent. Ces renseignements se rapportent notamment à des conditions de production respectueuses de l'environnement et du bien-être des animaux. Les déclarations concernant les produits OGM appartiennent au même chapitre. Cela améliorera la transparence et le fonctionnement du marché sans qu'il soit nécessaire de mettre en place des barrières douanières de nature technique. Un troisième type d'accords portera sur des critères économiques, écologiques et sociaux, en ce sens que l'on évitera de prendre des mesures commerciales qui auraient des répercussions négatives sur ces aspects. II convient enfin d'internaliser les coûts externes que peuvent comporter certaines méthodes de production agricole.

4) En ce qui concerne les trois piliers traditionnels - accès au marché, soutien interne et concurrence à l'exportation -, il convient simultanément d'en négocier les règles, les disciplines et les critères chaque fois que l'on envisage de nouvelles mesures de réduction.

5) Les négociations relatives à l'accès au marché doivent être basées sur le principe des concessions réciproques. On accordera des conditions d'accès spéciales aux pays en développement les plus pauvres. En ce qui concerne les contingents tarifaires, le choix du mode d'allocation doit rester une affaire interne pour autant que l'on respecte le principe de l'accès garanti. II convient de maintenir la clause de sauvegarde spéciale prévue pour l'agriculture, basée sur les volumes et les prix. La Suisse propose des améliorations concrètes à cet égard.

6) Du côté du soutien interne, les trois boîtes ${ }^{b}$ - orange, bleue et verte - devront être définies de manière plus précise que jusqu'à présent. Dans le domaine de la boîte verte, il appartient à chaque pays de déterminer le volume des prestations qu'il entend obtenir de son agriculture, ce qui revient à rejeter ici le principe du plafonnement. II s'agit d'indemniser le travail d'une agriculture multifonctionnelle dans les proportions voulues par le pays concerné, pour des prestations telles que l'entretien du paysage rural, I'occupation décentralisée du territoire et la préservation des ressources naturelles, de même que la sécurité des denrées alimentaires, le stockage à des fins d'approvisionnement du pays et le bien-être des animaux domestiques.

7) Les négociations sur la concurrence à l'exportation doivent englober non seulement les subventions directes, mais tous les types de programmes dans ce domaine - par exemple crédits à l'exportation, commerce d'Etat ou systèmes de prix mixtes. Et l'aide alimentaire ne doit pas servir abusivement à liquider des excédents.

Source: Document G/AG/NG/W/94; voir <www.wto.org > ou <www.blw.admin.ch〉.

a. Les pays en développement dans leur ensemble ont pu accroître leurs exportations agricoles en Suisse de 1 milliard de francs en 1995 à 1,2 milliard en 1999 (valeurs nominales). Leur part du marché suisse a augmenté dans des proportions encore plus fortes - de $10 \%$ en 1990 à $11 \%$ en 1995 puis à $16 \%$ en 1999 - du fait que le rendement brut épuré a régressé en Suisse de 8,8 milliards de francs en 1990 à 7,3 milliards en 1995.

b. L'Accord de I'OMC sur l'agriculture classe le soutien interne de ce secteur en différentes catégories, en fonction des effets qui en découlent sur la production et le commerce (d'après Senti [2000 : 481]):

- La boîte orange (réduction de $20 \%$ ) comprend les mesures de soutien spécifiques pour certains produits - suppléments, prix imposés, garantie de prise en charge et de prix, transferts imposés aux consommateurs en faveur de la production. 
- La boîte bleue (pas de réduction, mais nature de ces subventions à vérifier après l'échéance de la clause de paix fin 2003) englobe les paiements directs dans le cadre de programmes destinés à restreindre la production, lorsque ces paiements se rapportent à des surfaces et des rendements déterminés pour $85 \%$ au maximum de la production de base, ou si les primes versées pour le bétail se rapportent à un cheptel fixe.

- La boîte verte (pas de réduction, augmentations et nouveaux instruments possibles en tout temps) correspond aux mesures de soutien interne qui ont une influence nulle ou faible sur le commerce et la production, en recourant exclusivement aux deniers publics (transferts sur les consommateurs non autorisés). L'annexe 2 de l'Accord sur l'agriculture mentionne les exemples suivants: subsides pour la recherche, formation, vulgarisation, contrôle, informations sur le marché, infrastructures, stockage par les pouvoirs publics, aide alimentaire intérieure, aides directes au revenu, programmes de garantie des revenus, paiements lors de catastrophes naturelles, programmes incitant des producteurs à cesser leurs activités, retrait de ressources de la production, aides à l'investissement, programmes de subventions écologiques et régionales.

\subsection{LA PHASE DE MISE AU POINT (MARS 2001-MARS 2002)}

La «séance d'inventaire» du 28 mars 2001 s'est appuyée sur les 48 propositions émises jusque-là pour établir le menu de la deuxième phase. Chaque participant aux négociations a jusqu'à mars 2002 pour présenter le détail de ses idées. Voici la liste des sujets débattus:

- accès au marché (droits de douane, contingents tarifaires et méthodes d'allocation, clause de sauvegarde spéciale);

- concurrence à l'exportation (y compris crédits à l'exportation, aide alimentaire à crédits subventionnés, commerce d'Etat et restrictions aux exportations);

- soutien interne (boîtes orange, verte et bleue);

- sécurité de l'approvisionnement;

- sécurité des denrées alimentaires;

- développement rural;

- indications géographiques;

- environnement;

- questions de développement (préférences tarifaires, importateurs de produits alimentaires, monocultures, petits pays insulaires, coopération technique);

- information des consommateurs, labels;

- initiatives sectorielles (p. ex. franchise douanière pour les spiritueux sur les principaux marchés).

\subsection{RELATIONS AVEC D'AUTRES NÉGOCIATIONS}

Les négociations dont il est question ici se concentrent clairement sur les «trois piliers » (accès au marché, soutien interne et concurrence à l'exportation) ainsi que - selon le point de vue suisse - sur la multifonctionnalité. Mais il convient de prendre également en considération toute une série d'autres dossiers qui présentent des liens plus ou moins étroits avec les négociations agricoles de l'OMC et revêtent une importance pour l'avenir des politiques adoptées en matière d'agriculture et de développement. Cela met également en lumière la complexité de ces pourparlers, qu'il est bien entendu impossible de ramener à une formule simple. 
$\square$ Parmi les accords de l'OMC: ADPIC, OTC, etc.

La Suisse estime que certaines règles des accords conclus dans le cadre de l'OMC devront être modifiées, complétées ou réinterprétées pour répondre au critère complexe de la multifonctionnalité. Deux de ces accords revêtent à cet égard une importance particulière:

- l'Accord sur les aspects des droits de propriété intellectuelle qui touchent au commerce (ADPIC), dont les dispositions devront être étendues à des biens immatériels tels que les indications géographiques ${ }^{15}$;

- l'Accord sur les obstacles techniques au commerce (OTC) ${ }^{16}$ devra spécifier dans quelles conditions des déclarations relatives aux méthodes de production ou à des normes concernant le bien-être des animaux pourront être exigées.

Mais les principes applicables aux subventions en général, le droit relatif aux investissements et à la concurrence (encore embryonnaire), les accords de libreéchange et unions douanières méritent eux aussi une attention particulière dans le contexte agricole et, bien entendu, dans celui de la politique de développement, du fait que ces règles exercent la fonction essentielle de garantir des conditions de concurrence aussi équitables et exemptes de distorsions que possible tout en tenant compte des spécificités de l'agriculture.

Il va de soi que la problématique commerce-environnement concerne tout particulièrement le secteur agricole. Il s'agira de déterminer si les écarts de compétitivité résultant de normes écologiques différentes revêtent une importance économique suffisante pour qu'il faille intervenir dans le sens d'une harmonisation (ou d'un mécanisme de compensation). On reproche souvent aux pays en développement de pratiquer un «dumping écologique», mais cette accusation n'a guère été étayée par des chiffres concrets - tout au moins pour les principaux produits commercialisés que sont les céréales, les oléagineux et la viande ${ }^{17}$.

$\square$ Hors OMC: CDB, Cartagena, EI, Convention sur le climat, Kyoto, $F A O$, Codex alimentarius, IPPC ${ }^{18}$, OIE, OIT

Toute une série d'autres accords et organisations ont leur rôle à jouer dans ce contexte; nous n'en donnons ici qu'un bref aperçu.

La Convention sur la diversité biologique et la Convention sur les changements climatiques, signées l'une et l'autre en 1992, revêtent une importance considérable pour le commerce agricole et la politique intérieure en la matière.

\section{Accords hors OMC}

La Convention sur la diversité biologique (CDB) est un élément central des efforts internationaux pour préserver la biodiversité en tant qu'héritage collectif de l'humanitéa. La CDB poursuit les trois grands objectifs suivants: conservation des ressources génétiques; utilisation durable de ces ressources; répartition équitable et équilibrée des avantages résultant de leur exploitation. Les ressources génétiques au sens où l'entend cette convention se rapportent à toutes les formes de vie de notre planète

15. RS [Recueil systématique du droit fédéral] 0.632.20/RO 1995, p. 2457 (cf. en particulier art. 22-24).

16. Ibid., p. 2244.

17. Cf. Grote et al. (2001: 240 sqq.).

18. IPPC: Integrated Pollution Prevention and Control (en français PRIP: Prévention et réduction intégrées de la pollution). 
(animaux et végétaux aquatiques, terrestres et aériens). La notion de diversité agrobiologique correspond aux ressources génétiques de l'agriculture et de l'alimentation, avec les plantes et les animaux traditionnellement utilisés ainsi que les espèces sauvages importantes pour l'alimentation.

En janvier 2000, environ 140 Etats ont adopté à l'unanimité le Protocole de Cartagena sur la prévention des risques biotechnologiques, qui fait également partie de la $\mathrm{CDB}$. Ce document règle le commerce international d'organismes génétiquement modifiés (OGM): tout pays a le droit de refuser l'importation d'OGM vivants en cas de doutes justifiés concernant leur innocuité pour l'homme et l'environnement. On se base ici sur le principe de précaution, ce qui est nouveau pour une convention internationale (cf. encadré suivant).

L'Organisation des Nations Unies pour l'alimentation et l'agriculture (FAO) s'est intéressée dès la fin des années 1960 aux questions de conservation, d'échange et d'utilisation des ressources génétiques. La sécurité à long terme de la population mondiale en matière d'alimentation, l'adaptabilité de la nature et les solutions à trouver pour assurer la sécurité de l'approvisionnement en produits agricoles sont fortement tributaires de la diversité génétique. L'Engagement international sur les ressources phytogénétiques (EI), signé en 1983 sans imposer de contrainte juridique, se situe à la charnière entre agriculture, commerce et environnement. Accéder aux ressources génétiques pour l'agriculture et l'alimentation, répartir équitablement les avantages que procure l'exploitation des ressources génétiques, reconnaître les apports historiques et actuels des paysans pour la conservation de la diversité génétique nécessaire à l'alimentation et à l'agriculture, et assurer l'approvisionnement des populations en denrées alimentaires comptent parmi les objectifs essentiels que doivent poursuivre ces trois domaines. On s'efforce ainsi depuis 1994 d'harmoniser l'El avec la Convention sur la diversité biologique. Un des points importants est la liste des plantes librement disponibles, voire la répartition équitable (entre pays en développement et pays industrialisés) des avantages résultant de leur utilisation.

Cette problématique englobe par ailleurs le privilège de l'agriculteur et le privilège de l'éleveur ${ }^{\text {b }}$. Ces deux principes ne sont pas incontestés. II est fréquent, notamment, que le premier soit refusé aux agriculteurs dans les contrats de culture et de prise en charge passés avec des entreprises de semences et de commercialisation.

Dans le cadre du Protocole de Kyoto découlant de la Convention sur les changements climatiques, dont les dispositions d'exécution ont été adoptées par les parties le 27 juillet 2001 à Bonnc, la Suisse s'est engagée à réduire entre 2008 et 2012 ses émissions de six gaz à effet de serre dans la proportion de $8 \%$ en équivalents $\mathrm{CO}_{2}$ par rapport à 1990. Un des sujets à la fois les plus disputés à Bonn et les plus importants pour l'agriculture était de savoir si l'on a le droit de prendre en considération les puits (propriété naturelle de la biosphère à absorber $\mathrm{du} \mathrm{CO}_{2}$ ). D'une manière générale, toute extensification de la production agit positivement sur la situation globale de l'écosystème (qualité des sols, risque d'érosion, biodiversité, protection des eaux souterraines, etc.). De plus, il y a maintenant la possibilité de considérer comme puits des surfaces agricoles converties en forêts. Selon les premières estimations, la prise en compte de ces surfaces pourrait représenter environ un quart de la diminution obligatoire des émissions suisses en vertu du Protocole de Kyoto. Cependant, le processus de reboisement (environ 6000 ha par année entre 1985 et 1995) est en contradiction avec les objectifs de la politique agricole suisse (maintien des surfaces cultivées). Le potentiel "puits" pourra être encore accru par des méthodes de culture telles que le non-labour ou la conversion de champs cultivés en prairies (surfaces de compensation écologique) et en forêts.

a. La Suisse a ratifié la Convention de 1992 sur la diversité biologique (entrée en vigueur le 19 février 1995, RS 94.040).

b. Le privilège de l'agriculteur autorise celui-ci à réutiliser les semences issues de la récolte pour son propre usage, même s'il s'agissait au départ de semences achetées. Cette pratique est importante pour la sécurité alimentaire et l'autonomie économique des paysans.

Le privilège de l'éleveur lui donne accès aux variétés végétales ou aux races animales qui pourront donner lieu à de nouveaux croisements par l'échange de ressources génétiques.

Cf. Andrea Arz de Falco, "Umstrittene Patente auf Tiere und Pflanzen", Neue Zürcher Zeitung, 28.8.01, p. 14.

c. Le compromis de Bonn figure dans la Décision 5/CP-6 Mise en œuvre du Plan d'action de Buenos Aires (FCCC/CP/2001/ L7), <www.unfccc.int/resource/docs/cop6secpart//07.pdf >. On trouve également un résumé des négociations et des résultats de Bonn dans le Earth Negotiations Bulletin, <www.iisd.ca/linkages/vol12/enb12176e.html〉. 
L'évolution des exigences en matière d'hygiène et celle du commerce international de produits agricoles confèrent également une importance croissante à la nécessité d'harmoniser les normes relatives aux denrées alimentaires et à d'autres produits. Les principales instances de normalisation sont le Codex alimentarius (OMS/FAO) $)^{19}$, la Convention internationale pour la protection des végétaux (CIPV) ${ }^{20}$ et l'Office international des épizooties (OIE) ${ }^{21}$. Les accords de l'OMC confèrent une importance accrue au travail de ces organisations (que l'on surnomme les «trois sœurs»). En effet, l'Accord sur l'application des mesures sanitaires et phytosanitaires (Accord SPS) ${ }^{22}$ a instauré de nouvelles règles juridiques en matière de commerce agricole: le non-respect de ces normes universelles n'est désormais admis que si l'argument d'un risque accru (justifiant des critères de production ou d'admission plus sévères) est étayé par des preuves scientifiques.

\section{Le principe de précaution}

La libéralisation croissante du commerce agricole confère une importance d'autant plus grande à la sécurité des denrées alimentaires. Pour tout pays, la protection des consommateurs (protection de la santé et contre les tromperies) est une préoccupation légitime et incontestée dans son principe. Il en va de même pour la protection de l'environnement. Un problème de politique commerciale se pose au moment où l'évaluation et la gestion des risques sont rendues difficiles par un manque de données scientifiques. Dans ce genre de situation, le principe de précaution permet à un pays de définir luimême un niveau de protection approprié, même sans directives internationales (ou de déroger à cellesci pour autant que l'on dispose de preuves justificatives scientifiques suffisantes, cf. art. 5 SPS).

On utilise dans ce contexte la notion de traçabilité ("de la fourche à la fourchette») pour localiser et réduire les dangers potentiels. Cette forme de gestion des risques permet également d'évaluer diverses méthodes de production quant à la sécurité du produit final, et de déterminer le «niveau de protection approprié" (cf. Accord SPS).

L'affaire de la viande aux hormones (Etats-Unis/Canada contre UE), en particulier, conduit à réévaluer

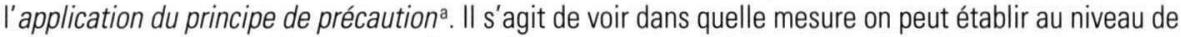
I'OMC des principes juridiques garantissant la protection de la santé sans protectionnisme économique. On devrait ainsi examiner, en marge des nouvelles négociations agricoles, si l'Accord SPS correspond à la situation actuelle.

a. Cf. European Communities: Measures concerning Meat and Meat Products (Hormones), Report of the Appellate Body, WT/DS26/AB/R, WT/DS48/AB/R, 16 January 1996

L'ouverture des marchés relance également la question des normes sociales (en particulier les disparités des conditions de travail). Ce problème se pose non pas au niveau des salaires en valeur absolue, mais à celui des distorsions de la concurrence provoquées par les pratiques exercées dans de nombreux pays - travail obligatoire (notamment dans l'agriculture), travail des enfants ou

19. Instauré le 4 novembre 1961 à Rome. Tous les membres de l'OMS ou de la FAO sont automatiquement membres døu Codex alimentarius. Des représentants de l'économie privée prennent une part active aux travaux du Codex en qualité d'observateurs et d'experts techniques; consommateurs et ONG y sont encore peu représentés.

20. Du 6 décembre 1951 (RS 0.916.20).

21. Arrangement international du 25 janvier 1924 pour la création, à Paris, d'un office international des épizooties (RS 0.916.40).

22. RO 1995, p. 2178. 
absence de liberté syndicale et d'accords collectifs. La Suisse considère que ces questions doivent être traitées non par l'OMC, mais par l'Organisation internationale du travail (OIT). Toutefois, l'application de ces normes au commerce international s'est heurtée jusqu'à présent à une résistance acharnée de presque tous les pays en développement, lesquels y voient - pas toujours à tort - des manœuvres protectionnistes de certains pays industrialisés. Il se pourrait bien que l'approche pragmatique de l'OIT constitue une alternative valable à une clause sociale de l'OMC qui ne tiendrait pas compte des divers niveaux de développement ${ }^{23}$. La Suisse, qui a ratifié l'ensemble des normes de travail fondamentales de l'OIT $^{24}$, plaide pour une clarification des relations entre ces normes et les règles commerciales; quoique insuffisant, le compromis obtenu à Doha constitue après Seattle le meilleur résultat que l'on pouvait atteindre par consensus ${ }^{25}$.

\subsection{LE RÉSULTAT DE LA CONFÉRENCE MINISTÉRIELLE DE DOHA}

\section{$\square$ En général}

Après une «dernière nuit» marathon de trente-six heures, la Conférence a adopté une déclaration ministérielle annonçant un cycle complet de négociations, «l'agenda de développement de Doha». Cette résolution renforce l'Organisation mondiale du commerce, qui se trouve ainsi remise en selle après l'échec de Seattle et en dépit (ou à cause) des attentats du 11 septembre 2001. Mais les négociations proprement dites et les principaux défis à relever attendent encore d'être empoignés!

\section{$\square$ Agriculture}

Dans le secteur agricole, la conférence confirme l'obligation de «poursuivre le processus de réforme» déjà contenue dans l'article 20 de l'Accord de l'OMC sur l'agriculture - quoiqu'en donnant un certain nombre de précisions et d'échéances.

Le texte adopté à Doha concernant l'agriculture (en six phrases seulement!) est l'exemple type d'un mandat de négociation «suffisamment imprécis ${ }^{26}$. Il n'a

23. Cf. Martin Senti, «Soziale Rechte und wirtschaftliche Öffnung », Neue Zürcher Zeitung, 8.9.01, p. 90.

24. La Déclaration de l'OIT relative aux principes et droits fondamentaux au travail (1998) comprend les dispositions suivantes: interdiction du travail forcé ( $\mathrm{n}^{\circ \mathrm{s}} 29$ et 105), élimination de la discrimination ( $\mathrm{n}^{\mathrm{os}} 100$ et 111), abolition du travail des enfants ( $\mathrm{n}^{\mathrm{os}} 138$ et 182), liberté d'association et droit de négociation collective ( $\mathrm{n}^{\mathrm{os}} 87$ et 98 ).

25. «Par: 8: Nous réitérons la déclaration que nous avons faite à la Conférence ministérielle de Singapour concernant les normes fondamentales du travail internationalement reconnues. Nous prenons note des travaux en cours à l'Organisation internationale du travail (OIT) sur la dimension sociale de la mondialisation. »

26. «Par. 13. Nous reconnaissons les travaux déjà entrepris dans les négociations engagées au début de 2000 au titre de l'article 20 de l'Accord sur l'agriculture, y compris le grand nombre de propositions de négociation présentées au nom de 121 Membres au total. Nous rappelons l'objectif à long terme mentionné dans l'Accord, qui est d'établir un système de commerce équitable et axé sur le marché au moyen d'un programme de réforme fondamentale comprenant des règles renforcées et des engagements spécifiques concernant le soutien et la protection afin de remédier aux restrictions et distorsions touchant les marchés agricoles mondiaux et de les prévenir. Nous reconfirmons notre adhésion à ce programme. Faisant fond sur les travaux accomplis à ce jour et sans préjuger du résultat des négociations, nous nous engageons à mener des négociations globales visant à: des améliorations substantielles de l'accès aux marchés; des réductions de toutes les formes de subventions à l'exportation, en vue de leur retrait progressif; et des réductions substantielles du soutien interne ayant des effets de 
d'ailleurs subi à Doha même qu'une seule modification, l'UE ayant réussi à imposer une atténuation de l'objectif final visé par la suppression des subventions à l'exportation. Ce mandat constitue toutefois un signal politique et aura sans aucun doute des effets sur la suite des négociations agricoles: on est censé définir pour mars 2003 les «modalités» d'établissement des listes de concessions, à savoir les formules de réduction des tarifs douaniers, l'augmentation des contingents tarifaires, la redéfinition des formes de soutien interne et des règles applicables, et - ce qui sera probablement le plus difficile - la limitation et la réduction de toutes les formes de subventions à l'exportation; et tout cela compte tenu de la problématique du développement et de celle de la multifonctionnalité !

\section{$\square$ Evaluation du point de vue de la Suisse}

Le résultat de ces négociations n'impose pas de contraintes à la Suisse et lui permet de poursuivre sa politique agricole (PA 2007, cf. sous-chapitre 3.4) conformément à son mandat constitutionnel. C'est un fait aussi que l'on ne conteste plus le rôle multifonctionnel de l'agriculture, de sorte que l'objectif principal de la Suisse en matière agricole a été entièrement atteint.

La formulation du mandat de négociation relatif aux aspects multifonctionnels de l'agriculture correspond aux dispositions convenues à l'article 20. Ce mandat reste ainsi en deçà de la solution idéale préconisée par la Suisse, mais s'avère néanmoins acceptable en ce qu'il ne bloque rien. Il sera cependant indispensable de renouveler et d'intensifier les efforts pour faire comprendre et accepter ces préoccupations, en particulier au niveau des instruments d'application (dans le cadre de l'Accord sur l'agriculture: boîte verte; en dehors: indications géographiques, labels, principe de précaution). Si l'on n'y parvenait pas, la «prise en considération des préoccupations autres que commerciales» confirmée à Doha ne débouchera guère sur des négociations significatives.

\section{LA POLITIQUE AGRICOLE SUISSE ET LES DÉFIS DE L'OMC}

Les profondes réformes intervenues au cours des dix dernières années ont bouleversé l'agriculture suisse. Il y a fallu entre autres plusieurs votations populaires. Où en est la politique agricole suisse aujourd'hui, sept ans après l'entrée en scène de l'OMC?

distorsion des échanges. Nous convenons que le traitement spécial et différencié pour les pays en développement fera partie intégrante de tous les éléments des négociations et sera incorporé dans les Listes de concessions et d'engagements et selon qu'il sera approprié dans les règles et disciplines à négocier, de manière à être effectif d'un point de vue opérationnel et à permettre aux pays en développement de tenir effectivement compte de leurs besoins de développement, y compris en matière de sécurité alimentaire et de développement rural. Nous prenons note des considérations autres que d'ordre commercial reflétées dans les propositions de négociation présentées par les Membres et confirmons que les considérations autres que d'ordre commercial seront prises en compte dans les négociations comme il est prévu dans l'Accord sur l'agriculture.

Par. 14. Les modalités pour les nouveaux engagements, y compris les dispositions pour le traitement spécial et différencié, seront établies au plus tard le 31 mars 2003. Les participants présenteront leurs projets de Listes globales fondées sur ces modalités au plus tard à la date de la cinquième session de la Conférence ministérielle. Les négociations, y compris en ce qui concerne les règles et disciplines et les textes juridiques connexes, seront conclues dans le cadre et à la date de la conclusion du programme de négociation dans son ensemble.» 


\subsection{UN CHANGEMENT D'ORIENTATION SUSCITÉ DE L'EXTÉRIEUR: OMC, UE}

L'adoption des obligations inhérentes à l'Accord de l'OMC sur l'agriculture ${ }^{27} \mathrm{a}$ entraîné entre 1995 et 2000 la transformation du régime de protection douanière: remplacement des restrictions à l'importation par des droits de douane, conversion des quantités importées en 1986-1988 en contingents bénéficiant de bas tarifs, réduction des tarifs de $15 \%$ au minimum et de $36 \%$ en moyenne arithmétique. Parallèlement à ses mesures de réforme internes, la Suisse a fait, à bien des égards, mieux que remplir ses engagements en matière de soutien interne $(-20 \%)$ et de subventions à l'exportation $(-21 \%$ des quantités, $-36 \%$ des montants) ${ }^{28}$.

L'Accord entre la Communauté européenne et la Confédération suisse relatif aux échanges de produits agricoles du 21 juin $1999^{29}$ (un des sept accords sectoriels entre la Suisse et l'UE) entrera probablement en vigueur au cours du premier semestre 2002. Il apportera par étapes la franchise douanière du commerce avec l'UE pour le fromage et certaines cultures spéciales (fruits, légumes, spécialités de vins et de viandes). Il en résulte toutefois un préjudice pour les produits correspondants des pays en développement, dans la mesure où ceux-ci ne bénéficient pas de préférences tarifaires ${ }^{30}$.

\subsection{LA RÉFORME INTÉRIEURE ET SES EFFETS: PA 2002}

Vu sous l'angle de l'OMC et des règles que celle-ci a mises en vigueur, ce changement d'orientation représente une performance considérable. Mais l'élément déclencheur de la réforme agricole a été d'ordre intérieur - en particulier pour ce qui concerne la suppression de toutes les garanties de prix et d'écoulement données par l'Etat ainsi que le report d'une grande partie du soutien du marché en paiements directs indépendants de la production et assortis de conditions écologiques (donc non soumis aux obligations de réduction):

- les dépenses de soutien du marché ont régressé, dans le budget de la Confédération consacré à l'agriculture, de $67,0 \%$ en 1990 à $28,1 \%$ en 2000 ; selon le plan financier, elles se chiffreront à $22,6 \%$ en 2003 . Inversement, les paiements directs passent de $21,9 \%$ en 1990 à $65,6 \%$ en 2000 et à $69,6 \%$ en $2003^{31}$;

- la nouvelle loi fédérale sur l'agriculture du 29 avril 1998 (LAgr; RS 910.1) et la politique agricole 2002 qui en découlait ont encore accéléré cette évolution. On a introduit en même temps deux nouveaux instruments de la politique agricole destinés à obtenir de meilleurs prix sur des marchés libéralisés: la protection des indications géographiques (p. ex. appellations

27. RO 1995, p. 2150.

28. Cf. Office fédéral de l'agriculture (2000 et 2001, op. cit.).

29. FF 1999, p. 4645; cf. aussi Message du 23 juin 1999 relatif à l'approbation des accords sectoriels entre la Suisse et la CE, pp. 98-126 (99.028).

30. Par contre, les 48 pays les moins avancés bénéficient dès le 1er janvier 2002 de la première de trois étapes devant aboutir à l'accès au marché suisse sans droits de douane (ni contingents) pour tous leurs produits.

31. La différence par rapport aux $100 \%$ correspond aux dépenses pour amélioration des bases de production (sources: 7e rapport sur l'agriculture; Message concernant un arrêté fédéral sur les moyens financiers destinés à l'agriculture pour les années 2000-2003 du 18 novembre 1998 (FF 1998, p. 503). 
d'origine contrôlée) $)^{32}$ ainsi que la désignation officielle de divers modes de production tels que l'agriculture biologique ${ }^{33}$.

Ces réformes ont eu leurs effets: les exploitations agricoles évoluent dans le sens d'unités compétitives. L'évolution structurelle s'est légèrement accélérée dans les années 1990 (à 2,7\%), mais sans dépasser celle des années 1950 et $1960^{34}$. Cependant, les exploitations à titre principal sont toujours nettement plus petites (17 ha) qu'en Allemagne par exemple (41 ha). Les prix à la production ont par contre fortement baissé, à savoir d'environ 30\% entre 1990 et 1999 pour les produits principaux que sont le lait et le blé. Ces deux marchés ont été libéralisés sur le plan intérieur; les contingents laitiers, par exemple, ont été rendus négociables (1999) et augmentés de $3 \%$ (2001). Les prix se sont stabilisés depuis lors. Le revenu net de la main-d'œuvre familiale a même augmenté, mais se situe toujours à un bas niveau ${ }^{35}$. Ce sont d'ailleurs moins les consommateurs que l'industrie et le commerce de détail qui ont profité de la diminution des prix à la produc$\operatorname{tion}^{36}$.

\subsection{CRITIQUES ÉMISES PAR LES ORGANISATIONS INTERNATIONALES: OMC, OCDE, FMI}

Même réformée, la politique agricole actuelle n'échappe pas à certaines critiques, en particulier dans les instances internationales où la Suisse (comme tous les autres pays) doit se soumettre à des examens périodiques (cf. encadré ci-dessous).

\section{La politique agricole suisse vue par I'OMC, I'OCDE et le FMI}

La politique agricole suisse et sa politique du commerce extérieur dans ce domaine prennent une place importante dans le rapport de I'OMC (2000); les rapports sur les pays de I'OCDE (2000) et surtout du FMI (IMF 2001) traitent l'agriculture de façon plutôt succincte.

\section{Evaluation générale}

Pour toutes ces organisations, la nouvelle loi sur l'agriculture entrée en vigueur au début de 1999 n'empêche pas ce secteur de rester parmi les plus protégés qui soient. Les réformes instaurées jusqu'à présent ne constituent ainsi qu'un début, quoique apprécié (FMI).

Jugement positif(OCDE): les réformes récentes constituent un pas important vers la déréglementation du marché intérieur, en ce sens que a) la suppression des prix minimums et des marges de transformation garantis laisse mieux jouer l'offre et la demande sur le marché indigène des produits alimentaires d'origine agricole, et $b$ ) le réaménagement des paiements directs a pour effet que les paysans prennent leurs décisions de production en fonction des signaux du marché plutôt que des paiements qu'ils obtiennent des pouvoirs publics.

32. LAgr art. 15; Ordonnance du 28 mai 1997 concernant la protection des appellations d'origine et des indications géographiques des produits agricoles et des produits agricoles transformés (ordonnance sur les AOP et les IGP, RS 910.12).

33. LAgr art. 16; Ordonnance du 22 septembre 1997 sur l'agriculture biologique et la désignation des produits végétaux et des denrées alimentaires biologiques (ordonnance sur l'agriculture biologique, RS 910.18).

34. Office fédéral de l'agriculture (2001).

35. Le revenu agricole 2000 (rendement brut moins coûts réels) s'élève en moyenne à 64'675 francs par exploitation, 17,7\% au-dessus du niveau des années 1997-1999. Le revenu du travail (revenu agricole moins intérêts calculés pour les fonds propres investis dans l'exploitation) par unité de maind'œuvre non salariée a progressé de 13,5\% pour atteindre 38'099 francs (communiqué de presse du 24.8.01 de la Station fédérale de recherches en économie et technologie agricoles, Tänikon).

36. Tiré de «Landwirte auf der Höhe des Wettbewerbs », Neue Zürcher Zeitung, 22.11.00, p. 16. 
Les organisations internationales critiquent le fait que la protection du secteur agricole reste considérable: selon elles, le soutien des producteurs par l'Etat reste le plus élevé des pays de l'OCDE avec $73 \%$ des rendements agricoles bruts de 1997 , contre $40 \%$ en moyenne pour I'OCDE et I'UE. Bien que les prix à la production aient modérément reculé en 1999, l'écart avec les prix de l'UE se maintient à un niveau plus ou moins constant depuis 1992; I'objectif déclaré de réduire cet écart de moitié à l'horizon 2002 ne pourra guère être atteint. L'OMC estime que les effets des réformes sur les prix sont limités par un certain nombre de facteurs: manque de concurrence dans différents secteurs (p. ex. sucre, huiles), mesures de soutien des prix et remplacement d'anciennes organisations de commercialisation par des institutions mandatées par l'Etat.

Le renforcement des paiements directs de prestations écologiques transforme la structure du soutien apporté à l'agriculture, mais sans en abaisser le niveau - même à moyen terme.

\section{Recommandations}

Les organisations internationales considèrent que des réformes plus poussées seront indispensables, dans l'intérêt bien compris de la Suisse elle-même tout en étant profitables à la communauté internationale:

๑ une libéralisation plus systématique rendrait le secteur plus compétitif; la pression qui en résulterait sur les prix intérieurs profiterait à l'économie du pays dans son ensemble;

• la libération de main-d'œuvre agricole contribuerait à atténuer la pénurie de main-d'œuvre dans d'autres secteurs;

- la suppression des barrières protectionnistes à l'égard des produits agricoles et un engagement de la Suisse visant à éliminer rapidement la protection du secteur agricole sur le plan international seraient également très précieux pour les pays en développement.

Source: Seco, Schweizer Wirtschaftspolitik im Urteil der internationalen Organisationen (non traduit), juin 2001.

\subsection{POURSUITE DE LA POLITIQUE AGRICOLE: PA 2007}

Arrêt égale recul - mais il ne s'agit pas de faire la révolution. L'évolution nécessaire de la politique agricole se fait par étapes quadriennales, au cours desquelles le Parlement traite les propositions du Conseil fédéral et accorde les crédits correspondants.

\section{Politique agricole 2007}

La consultation relative à la période 2004-2007 ("Politique agricole 2007») a été lancée le 21 septembre 2001. A la différence des réformes fondamentales de la dernière décennie, il s'agit, dans la présente révision, d'avancer sur la voie tracée et d'optimiser les mesures de politique agricole en fonction des objectifs à atteindre et de l'évolution des conditions générales. Un enjeu majeur sera d'améliorer encore la compétitivité de l'agriculture et du secteur alimentaire suisses dans un contexte de durabilité. Dans la perspective des défis futurs à relever, il est proposé d'adapter le train de mesures agricoles sur cinq axes:

1) préservation des parts de marché dans des conditions de concurrence accrue, notamment par un assouplissement complémentaire du marché laitier;

2) accroissement des performances entrepreneuriales des agriculteurs par un élargissement de leur marge de manœuvre;

3) maintien d'emplois dans le milieu rural par une meilleure adaptation des instruments de politique agricole aux objectifs de la politique régionale;

4) mesures d'accompagnement en vue d'une évolution structurelle socialement supportable;

5) renforcement de la confiance des consommateurs envers les denrées alimentaires par une promotion accrue de la qualité et de la sécurité, en tirant tout le parti possible des instruments de politique agricole destinés à promouvoir une utilisation durable des ressources naturelles. 


\section{5. ÉVALUATION}

Critiques et recommandations doivent être prises en compte dans le processus d'évolution de la politique agricole suisse, pour mieux satisfaire les exigences internationales (OMC, UE) mais aussi freiner utilement le tourisme de consommation $^{37}$. Grâce à la réforme déjà réalisée, l'agriculture helvétique n'affronte pas ces défis sans atouts. Les résultats des négociations à venir devraient nécessiter peu de nouveaux aménagements en Suisse, pour le soutien du marché notamment. De même, les subventions à l'exportation ont déjà été fortement diminuées pour la plupart des produits. Mais en ce qui concerne la protection douanière, les différences de prix par rapport à l'étranger restent élevées depuis 1992 - de sorte que des réductions des droits de douane et l'augmentation des contingents tarifaires prétériteraient la production indigène pour de nombreux produits ; celle-ci perdrait à chaque fois des parts de marché si elle ne parvenait pas à réaliser en même temps des gains de productivité ou à supporter des réductions de prix équivalentes aux allégements des contraintes douanières.

C'est pourquoi le développement parallèle des trois grands axes - réformes intérieures, UE, OMC - revêt une grande importance économique et politique. Il serait souhaitable (et plus facile) de donner une certaine avance aux réformes en les motivant par des considération de politique intérieure.

Ces réformes permettront également de mieux relever les défis posés par l'OMC et par l'UE, principal partenaire commercial de la Suisse. Il ne sera naturellement pas simple de convaincre le secteur agricole de la nécessité de réduire encore les protections douanières. Cependant, même notre agriculture axée sur le marché indigène a besoin d'une OMC forte; on évitera ainsi que les règles universelles ne cèdent la place à l'arbitraire et que le ton soit donné par les ministres des Finances des grandes puissances.

\section{CONFLIT D'OBJECTIFS ENTRE POLITIQUE DE DÉVELOPPEMENT ET POLITIQUE AGRICOLE}

Du côté de la politique de développement, que faut-il penser de l'Accord de l'OMC sur l'agriculture et de la politique agricole suisse, des effets de cette dernière sur le commerce avec les pays en développement, et de la position suisse dans les négociations agricoles de l'OMC? Ce chapitre montre qu'en dépit de nombreuses règles dérogatoires en faveurs des pays en développement, il y a conflit d'objectifs aussi bien au niveau des règles de l'OMC qu'entre les mesures prises par la Suisse dans le domaine agricole d'une part, et dans celui de la politique de développement d'autre part.

37. Par tourisme de consommation, on entend les achats de produits alimentaires dans les pays voisins que font régulièrement des personnes résidant en Suisse. Coop Suisse estime que ces achats totalisent 1,6 milliard de francs par année depuis 1997. Si l'on compare ce chiffre aux dépenses des ménages privés pour des denrées alimentaires (37,1 milliards en 1998), cela représente pour l'agriculture suisse une «perte» de $4,3 \%$. Des différences de prix plus faibles par rapport à l'étranger permettraient de "rapatrier» au moins une partie de la production et des ventes correspondantes. Source: Office fédéral de la statistique, communiqué de presse de septembre 2001, p. 9. 


\subsection{PRISE EN COMPTE DES INTÉRÊTS DU DÉVELOPPEMENT AU SEIN DE L'OMC}

Selon la doctrine généralement admise, le commerce extérieur contribue à accélérer le développement économique d'un pays. Cette expérience, les nations industrialisées aussi bien que les pays en développement avancés l'ont faite au cours des cinquante dernières années dans des proportions sans précédent. Mais la réalité vécue est tout autre pour la plupart des pays les moins avancés. C'est pourquoi il existe depuis de nombreuses années des règles destinées à sauvegarder les intérêts propres au développement dans le système du commerce mondial (cf. encadré ci-dessous).

\section{Traitement spécial et différencié (S\&D) dans le cadre du GATT et de I'OMC}

La "Déclaration du Cycle de Tokyo" de 1973 reconnaît pour la première fois "l'importance d'appliquer des mesures différenciées pour les pays en développement, de manière à les faire bénéficier d'un traitement spécial et plus favorable dans les domaines de négociation où c'est faisable $\Perp^{a}$. C'est sur cette base qu'a été adoptée en 1979 la clause d'habilitation, laquelle constitue l'une des deux dérogations permanentes à la clause de la nation la plus favorisée $e^{b}$ qui participe au fondement juridique du GATT pour le système de préférences généralisées (autorisant l'octroi aux pays en développement de conditions tarifaires plus favorables que pour les autres parties contractantes du GATT).

Actuellement, les accords de I'OMC (plus de 30) comportent pas moins de 97 dispositions $S \& D$ servant à tenir compte du degré de développement variable des membres de l'organisationc. A côté des préférences tarifaires déjà mentionnées, les principales concernent

a) de nouvelles facilités d'accès au marché (art. XXXVII GATT, art. IV AGCS);

b) la prise en considération de besoins spécifiques des pays en développement par exemple lors de la définition des normes techniques (art. 10 SPS, art. 15 Accord antidumping, etc.);

c) une souplesse accrue dans l'application des règles générales, par exemple le principe d'interdire les subventions à l'exportation de produits industriels (art. 27.6 Accord sur les subventions) ou les restrictions imposées aux volumes d'importation en cas de difficultés de la balance des paiements (art. XVIII GATT);

d) des délais de transition prolongés dans presque tous les accords; et

e) l'octroi d'une assistance technique pour la mise en œuvre.

Les pays les moins avancés bénéficient en outre de règles particulières dans tous ces domaines; il est admis d'une manière générale que ces pays ne sont amenés à contracter des obligations que dans la mesure où celles-ci sont compatibles avec les nécessités de leur développement et avec leurs capacités financières, administratives et institutionnelles ${ }^{d}$.

L'Accord sur l'agriculture prévoit une libération de l'obligation de réduire certaines subventions intérieures, ainsi que des réductions moins importantes des subventions à l'exportation (24\% au lieu de $36 \%)$, des mesures de soutien du marché (14\% au lieu de $20 \%$ ) et des droits de douane (10\% au lieu de $15 \%$, les pays les moins avancés étant totalement exemptés de cette obligation). Le délai de transition a également été prolongé pour les pays en développement (dix ans au lieu de six)e.

a. Citation originale en anglais.

b. La clause de la nation la plus favorisée est la principale disposition de non-discrimination de I'OMC (art. I GATT): elle prescrit que l'octroi d'une concession tarifaire à un pays s'étend automatiquement à tous les membres de l'OMC. Seules autres exceptions à cette règle (outre les préférences tarifaires): les unions douanières et les accords de libre-échange (art. XXIV GATT).

c. Aperçu complet dans le Document OMC WT/COMTD/W/77/Rev.1 du 21 septembre 2001 (132 pages).

d. Accord instituant I'OMC, art. XI:2 (RO 1995, p. 2124).

e. Cf. Document OMC MTN.GNG/MA/W/24 du 20 décembre 1993. 
On peut se demander dans quelle mesure ce traitement spécial porte ses fruits. Question difficile: bien qu'il y ait au total 97 dispositions destinées à tenir compte des différences dans le niveau de développement des membres de l'OMC, cela n'a pas eu des effets clairement décelables en faveur des pays en développement - à l'exception peut-être de certaines préférences tarifaires (cf. encadré ci-dessus). Au contraire, la revendication d'un statut spécial par les pays en développement s'est avérée d'une certaine manière contre-productive ${ }^{38}$.

Faut-il en conclure, après le demi-siècle de S\&D du GATT, que les dispositions spéciales prévues par l'OMC ne parviendront pas davantage à donner des impulsions au développement des pays les plus pauvres? C'est là un jugement pessimiste, qui devrait être nuancé. Il serait nécessaire de concentrer les mesures S\&D très concrètement sur les maillons faibles du développement, qui sont très variables selon les secteurs ou les régions; les pays industrialisés se montreraient alors mieux disposés à garantir des traitements spéciaux correctement ciblés. Mais le principe de 1' «auto-élection» suffit à contrecarrer une telle démarche: le «tigre» Singapour, dont le revenu annuel par habitant atteint 33'000 dollars, pourrait théoriquement revendiquer le statut de pays en développement aussi bien que le Ghana avec ses 390 dollars - qui le mettent juste audessus du groupe des pays les moins avancés ! ${ }^{39} \mathrm{Il}$ est clair que les pays industrialisés refuseraient l'octroi d'un traitement spécial aux plus riches des pays en développement. Et dans le secteur agricole, un traitement indifférencié a pour effet que même le Nordeste brésilien ne peut bénéficier d'une préférence commerciale de la part de l'OMC alors que des millions de paysans pauvres vivent dans cette région. Par ailleurs, chaque réduction globale des droits de douane diminue d'autant l'"avance» des pays jouissant de préférences tarifaires, ce qui permet d'affirmer que beaucoup de pays en développement se trouvent pris aujourd'hui dans un «piège du $S \& D$ ». Le seul moyen d'échapper à ce piège consiste à moduler le traitement spécial dans l'espace et le temps, en le réservant aux situations manifestes de sous- ou de mal-développement.

\subsection{UNE "BOÎTE DE DÉVELOPPEMENT» À L'OMC?}

Face au conflit d'objectifs dont nous avons parlé, les négociations en cours de l'OMC dans le domaine agricole ne peuvent que tenter de concilier au mieux les instruments de la politique agricole avec ceux de la politique de développement. L'accès sans droits de douane à des marchés exempts de subventions dans tous les pays industrialisés est une illusion: les miracles prennent encore plus de temps à se réaliser que les cycles de négociations de l'OMC !

De nombreux pays en développement ont présenté au cours de l'été 2000 la revendication à première vue logique de créer une boîte de développement (cf. encadré page suivante) qui regrouperait l'ensemble des instruments S\&D

38. Cf. Michalopoulos et World Bank (2000: 19): de nombreux pays en développement ont par exemple omis, dans le cadre du $8^{\mathrm{e}}$ cycle de négociations du GATT, de s'engager à des réductions des droits de douane sur leurs importations en contrepartie de l'accès à des marchés extérieurs pour leurs propres exportations. Par conséquent et dans l'ensemble, les réductions tarifaires du GATT des années 1960 et 1970 servaient assurément mieux les intérêts exportateurs des nations industrialisées que ceux des pays en développement.

39. Contrairement à l'OCDE et aux institutions de Bretton Woods, l'OMC ne classe pas les pays en développement dans différentes catégories juridiques ou financières. 


\section{Une « boîte de développement » dans l'Accord de l'OMC sur l'agriculture}

\section{$\begin{array}{lll}\text { Revendications }^{a} & \text { Situation actuelle } & \text { Appréciation }\end{array}$}

1) Ne pas limiter les dépenses publiques consacrées au soutien des prix des aliments de base.

2) Octroyer aux paysans les plus pauvres des crédits à taux préférentiels.
Limitation de ces dépenses à $10 \%$ du budget total consacré au soutien du marché.
Valable comme mesure de durée limitée pour des pays dont le plafond des mesures de soutien est bas ${ }^{\mathrm{c}}$.

Se justifie comme exception permanente si l'on définit "les plus pauvres" et surveille le programme.
3) Protéger les aliments de base menacés par des importations (clause de sauvegarde spéciale).

\section{Admis uniquement si tous les producteurs d'une région peuvent en bénéficier (art. 6.2).}

Bonne proposition si l'on donne une définition nuancée des "aliments de base" produits dont le Cycle d'Uruguay a transformé les obstacles au commerce en droits de douane (art. 5).
4) Admettre des augmentations des droits de douane justifiées par la sécurité de l'approvisionnement.
Seulement contre réductions tarifaires sur d'autres produits (art. XXVIII GATT).
Proposition valable (charge de la preuve?!) .
5) Ne pas réduire les droits de douane pour les aliments de base.
Les négociations d'accès aux marchés portent en principe sur tous les produits.
6) Interdire le dumping sous forme Toutes les subventions à l'exde subventions à l'exportation. portation ont un plafond.
Valable pour les pays en développement les plus pauvres.

Valable pour les pays en développement les plus pauvrese.

7) Ne pas limiter les subventions au transport et à la commercialisation.

Limitation de ces dépenses à Un pas dans la mauvaise direction! $10 \%$ du budget total consacré au soutien du marché.

8) Exporter les aliments de base (excédentaires) sans droits de douane.
Les négociations d'accès aux Peut se justifier. marchés portent sur tous les produits.
9) Aide alimentaire: a) admettre les subventions pour frais de stockage; b) ne pas imposer de restrictions aux mesures gouvernementales.
Seulement si "strictement ciblée " (annexe 2, chap. 4).
Acheter régulièrement au-dessus des prix à l'importation n'est ni utile ni acceptable.

a. Selon Green et al. (2001).

b. Les articles indiqués sans autre précision se rapportent à l'Accord sur l'agriculture.

c. II conviendrait alors de bien faire la distinction entre diverses situations concrètes: il ne faudrait pas par exemple que le riz de Thaïlande ou le sucre du Brésil puissent en profiter !

d. Mais un ravitaillement assuré (en permanence) par une autarcie complète sous protection douanière coûte cher à l'économie d'un pays!

e. Ceux-ci demandent cependant que l'on ne limite pas les crédits à l'exportation pour l'aide alimentaire ! 
envisageables pour le commerce agricole ${ }^{40}$. Mais le défaut rédhibitoire d'une telle approche, c'est qu'elle place tous les pays en développement dans une seule et même catégorie, laquelle évoluerait définitivement (?!) dans la direction opposée à celle que prennent les nations industrialisées: le «piège du S\&D" referme ses mâchoires! Cette "boîte de développement» comporte toutefois plusieurs propositions intéressantes, dont les perspectives de succès seraient d'autant plus grandes si leur mise à contribution se limitait aux producteurs qui en ont vraiment besoin. Le plus gros problème est d'établir une distinction (vérifiable) entre les paysans vraiment démunis (low income/resource poor) et ceux qui sont à même de produire dans les conditions offertes par le marché.

\subsection{LA POLITIQUE AGRICOLE DE LA SUISSE ET SA POLITIQUE DE DÉVELOPPEMENT}

L'impossibilité de concilier entièrement et simultanément politique agricole d'une part et politique du développement d'autre part n'est pas une exclusivité de l'OMC. Pour la Suisse également, ces deux catégories de préoccupations inscrites l'une et l'autre dans la Constitution fédérale ${ }^{41}$ - donnent lieu à un conflit d'intérêts: le fait de soutenir et de promouvoir l'agriculture suisse pénalise au moins en partie les exportateurs de produits agricoles de l'hémisphère Sud. Au niveau des instruments d'application, ce conflit se manifeste moins par le soutien financier de l'agriculture que par les droits de douane à l'importation, qui doivent tenir compte «des débouchés existant pour les produits suisses similaires ${ }^{42}$. Car un libre accès aux marchés serait pour les pays en développement un des facteurs de croissance les plus efficaces qui soient. Ce conflit persistera tant que la politique agricole inclura protection douanière et soutien du marché. Il importe peu, à cet égard, que le marché suisse ait un volume relativement faible pour les produits autres que tropicaux des pays en développement. De même, le fait que l'application de la clause de la nation la plus favorisée profite surtout aux grands fournisseurs de l'UE et d'Amérique du Nord et aux pays en développement performants (Brésil, Argentine) ne saurait justifier un refus de libéraliser le marché agricole pour des raisons de politique de développement.

Mais il y a encore d'autres problèmes à court et à moyen terme, même dans l'hypothèse d'un accès totalement libre aux marchés: le manque de compétitivité (rapport prix/prestation) de nombreux pays en développement par rapport à d'autres fournisseurs, ainsi que les subventions à l'exportation et les mesures de soutien du marché pratiquées par beaucoup de pays industrialisés. L'île Maurice et Cuba ne pourront pas nous livrer du sucre tant que l'UE accorde 80 francs de subvention à l'exportation pour chaque sac de $100 \mathrm{~kg}$. Et les bananes antillaises n'arriveront guère jusqu'à nous quand la production d'une île ne suffit pas à remplir tout un navire de réfrigération et de maturation. Et l'exemple des

40. « $[\ldots]$ the provisions of which would be geared in a manner so as to provide developing countries the required flexibility to enhance domestic production for domestic consumption and to take such other measures which may be necessary to protect the livelihood of their farmers ». Cf. Green et al. (2001). Cette boîte de développement est également décrite dans un «non paper » qu'un grand nombre de pays en développement ont soumis en mars 2001 au Comité agricole de l'OMC (G/AG/NG/W/14).

41. L'article 54, al. 2 cst. spécifie tout d'abord que «la Confédération s'attache à préserver l'indépendance et la prospérité de la Suisse» et ensuite qu'elle «contribue notamment à soulager les populations dans le besoin et à lutter contre la pauvreté ainsi qu'à promouvoir [...] la coexistence pacifique des peuples et la préservation des ressources naturelles».

42. LAgr art.17 (RS 910). 
bananes permet d'ailleurs également de démontrer à quel point les préférences tarifaires de l'UE tiennent peu compte des besoins de développement qui varient entre pays producteurs et à l'intérieur de ceux- $\mathrm{ci}^{43}$.

Il ne sert ainsi pas à grand-chose que la Suisse, durant le Cycle d'Uruguay et après celui-ci, ait supprimé ses droits de douane sur les produits tropicaux qui ne font pas une concurrence directe à la production indigène. Et l'abrogation progressive, à partir de 2002, de tous les droits de douane pour les pays les moins avancés ne sera profitable à ceux-ci que si leurs produits satisfont aux prescriptions sanitaires et phytosanitaires - alors que les exigences qualitatives vont toujours en augmentant pour la viande, les fruits et légumes ou encore les aliments du bétail par exemple!

\section{RÉSUMÉ ET CONCLUSION}

Après la débâcle de Seattle, les nouvelles négociations agricoles de l'OMC ont démarré en janvier 2000 comme il avait été convenu en 1994. Les positions initiales de 121 pays sont sur la table depuis mars 2001. Mais un véritable pas en avant ne sera possible que par des négociations globales dans le cadre de l'OMC. «L'agenda de développement de Doha» a été adopté par la $4^{\mathrm{e}}$ Conférence ministérielle (9-14 novembre 2001). Ce cycle de négociations devrait être achevé à fin 2004 déjà, et même en mars 2003 pour ce qui est des modalités régissant la prochaine étape de libéralisation du secteur agricole. Ces règles devront prendre en considération de façon équitable les revendications des pays en développement, mais aussi celles des représentants d'une agriculture «multifonctionnelle». Il est effectivement dans l'intérêt de tous les pays - ceux en développement compris - d'aboutir à une entente sur la prochaine étape de réforme internationale dans le domaine agricole: en cas de chute des prix sur le marché mondial et d'un regain d'interventions nationales, ce ne seront certainement pas les paysans des régions en développement qui sortiront vainqueurs d'une telle «course des ministres des Finances».

Encore peu ouverte aux préoccupations des pays en développement, la politique agricole suisse le deviendra davantage au moment où les subventions à l'exportation, la protection douanière et le soutien du marché diminueront - par exemple à la suite des négociations agricoles menées dans le cadre de l'OMC. Mais cela prendra du temps. Les pays en développement ont pu accroître leur part dans les importations agricoles de la Suisse au cours des années 1990, et les moins avancés devraient bénéficier progressivement de la suppression totale des droits de douane en leur seule faveur. Toutefois le conflit d'objectifs entre politique agricole et politique de développement subsiste: protection douanière et soutien de l'agriculture suisse sur le plan interne procurent à celle-ci les moyens d'une production durable conformément au mandat constitutionnel. Mais cela freine en même temps les importations de produits agricoles en provenance des nations industrialisées aussi bien que des pays en développement. Diverses mesures à prendre par la politique de développement permettront de neutraliser partiellement ce conflit, lequel sera en outre désamorcé par les opérations de 
« découplage» et de différenciation du soutien apporté à l'agriculture suisse. Les arbitrages nécessaires devront cependant avoir lieu dans le cadre des processus de décision politiques - ou bien résulteront de l'adoption des résultats des négociations internationales.

\section{BIBLIOGRAPHIE}

Auroi Claude et Schümperli Younossian Catherine (dir.), 2001, Le commerce durable. Vers de plus justes pratiques commerciales entre le Nord et le Sud, Genève: IUED.

Gibbs/UNCTAD : Special and Differential Treatment in the Context of Globalization, G15-Symposium, 10.12.98.

Green Duncan, CAFOD et Priyadarshi Shishir, 2001, Proposal for a "Development Box" in the WTO Agreement on Agriculture, Geneva: South Centre, June.

Grote Ulrike et al., 2001, "Vollkosten, Umweltstandards und internationale Wettbewerbsfähigkeit», Berichte über Landwirtschaft, Band 079, $\mathrm{n}^{\circ}$ 2, Berlin, Mai, pp. 234-250.

Häberli Christian, 2000, «The WTO Dispute Settlement Procedure: Implications for Agricultural Trade Arising from the Bananas Case», in Bilal Sanoussi et Pezaros Pavlos (eds.), Negotiating the Future of Agricultural Policies: Agricultural Trade and the Millennium WTO Round, Kluwer Law International, pp. 211-223.

Institut universitaire d'études du développement (IUED), 2001, Annuaire Suisse-Tiers Monde 2001, Genève: IUED.

International Monetary Fund, 2001, Switzerland: 2001 Article IV Consultation - Staff Report, Country Report, $n^{\circ}$ 01/74, May, <www.imf.org>.

Joerchel Anhorn Bastienne, 2001, «Les incohérences du commerce mondial. Le cas de la Banane», in Auroi Claude et Schümperli Younossian Catherine (2001: 27-40).

Lettington Robert J.L., 2001, «The International Undertaking on Plant Genetic Resources in the Context of TRIPs and the CBD», Bridges, International Centre for Trade and Sustainable Development, Year $5, \mathrm{n}^{\circ} 6$, July-August.

Michalopoulos C./World Bank, 2000, Trade and Development in the GATT and WTO: Role of S\&D for Developing Countries, document de travail pour le séminaire OMC du 10 mars 2000.

OCDE, 2000, Etudes économiques de l'OCDE, Suisse, Paris, décembre, librairie en ligne: <www.oecd.org/bookshop>.

Office fédéral de l'agriculture, 2000, Rapport agricole 2000, Berne.

-, 2001, Rapport agricole 2001, Berne.

OMC, 2000, Rapport final de l'examen de la politique commerciale de la Suisse, décembre, Dokument WT/TPR/S/77 vom 6. November 2000, <www.wto.org>.

Senti Richard, 2000, WTO - System und Funktionsweise der Welthandelsordnung, Zürich: Schulthess. 